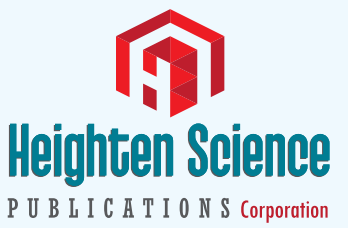

ISSN

2639-6629
*Address for Correspondence: Martin Rosas Peralta, "A Todo-Corazón" Program from IMSSMexico, and Head of research seminary at Postgrad program of "La Salle" Mexican Faculty of Medicine, Avenida Cuauhtémoc 330, Doctores, 06720 Cuauhtémoc, CDMX, Mexico City, Mexico, Email: mrosas_peralta@hotmail.com

Submitted: 16 January 2019

Approved: 25 January 2019

Published: 28 January 2019

Copyright: @ 2019 Ocampo LA, et al. This is an open access article distributed under the Creative Commons Attribution License, which permits unrestricted use, distribution, and reproduction in any medium, provided the original work is properly cited

Keywords: Diastolic dysfunction; HFpEF; Heart failure; Risk factors; Mexico

Check for updates

\title{
Heart Failure with preserved Ejection Fraction (HFpEF); A Mexican cohort from Mexican Institute of Social Security (IMSS)
}

\author{
Lizette Arizmendi-Ocampo ${ }^{1}$, Martin Rosas Peralta ${ }^{2 *}$, Daniela \\ Salinas Morales ${ }^{3}$, Alejandro Chanona Espinosa ${ }^{3}$, Alicia \\ Contreras Rodríguez ${ }^{4}$, Erick Ramírez Arias ${ }^{5}$, Carlos Riera \\ kinkel ${ }^{6}$, Guadalupe Castro Martínez ${ }^{7}$ and José Antonio \\ Magaña-Serrano ${ }^{7}$
}

${ }^{1}$ Social Service in Research, Mexican Faculty of Medicine from "La Salle", University of Mexico, Mexico City, Mexico

${ }^{2}$ A Todo-Corazón" Program from IMSS-Mexico, and Head of research seminary at Postgrad program of "La Salle" Mexican Faculty of Medicine of Mexico City, México ${ }^{3}$ Cardiology Department, Cardiology Hospital, CMN SXXI, IMSS, Mexico ${ }^{4}$ Echocardiography Department, Cardiology Hospital, CMN SXXI, IMSS, Mexico ${ }^{5}$ Emergency Department, Cardiology Hospital, CMN SXXI, IMSS, Mexico ${ }^{6}$ Director, Mexican Faculty of Medicine, "La Salle", Mexico City, Mexico ${ }^{7}$ Head of Heart Failure Division, Cardiology Hospital, CMN SXXI, IMSS, Mexico

\section{Abstract}

Background: Several epidemiologic studies indicate that up to $50 \%$ of patients with heart failure have a preserved ejection fraction, and this proportion has increased over time. The knowledge of its severity and associated comorbidity is determining factor to develop adequate strategies for its treatment and prevention. This study was focus on the creation of a cohort and follow-up of Mexican population and to analyze its severity as well as its interaction with the comorbidity of other cardiovascular risk factors.

Methods: We included patients from different sites of Mexico City than were sent to the Cardiology hospital of the National Medical Center in Mexico City for the realization of an echocardiogram as part of their assessment by the presence of dyspnea, edema, or suspicion of hypertensive heart disease. Complete medical history, physical examination and laboratory studies including Brain Natriuretic Peptide (BNP) serum levels were performed. Diagnosis of diastolic dysfunction was based on symptoms and echocardiographic data including time of deceleration, size of left atrium, e' septal and e' lateral, as well as $E$ wave, $A$ wave and its ratio $E / A$. All patients had left ventricle ejection fraction $>45 \%$.

Results: We included 168 patients with HFpEF. The most common risk factor was hypertension $(89.2 \%)$, followed by overweight and obesity $(>78.5 \%)$, dyslipidemia $(82.1 \%)$ and diabetes $(42.8 \%)$. Women were dominant, 108 (64.3\%); the mean age was 63 years old. When we classify by severity of diastolic dysfunction, we found that $41.1 \%$ were grade I, $57.1 \%$ were grade II and only $1.8 \%$ were grade III. The risk factors most strongly associated with the severity of diastolic dysfunction were hypertension, obesity and dyslipidemia. We found BNP levels highly variables, but the levels were higher detected as the ejection fraction was approaching to $45 \%$. At one year of follow up mortality was not reported.

Conclusion: HFpEF is a frequent entity in patients with cardiovascular risk factors in Mexico. The most common risk factor was hypertension. The combination of hypertension, overweight and dyslipidemia predicted the severity of diastolic dysfunction. We recommend that all Mexican patient with hypertension and overweight or obesity should be submitted as a part of its medical evaluation to an echocardiogram study in order to detect diastolic dysfunction even though the signs or symptoms are or not evident. 


\section{Introduction}

Several epidemiologic studies indicate that up to $50 \%$ of patients with heart failure have a preserved ejection fraction, and this proportion has increased over time [1]. In observational studies, rates of hospitalization and death among patients who have heart failure with a preserved ejection fraction are similar with those patients who have heart failure with a reduced ejection fraction [1], but in clinical-trial populations, outcomes are better in patients who have heart failure with a preserved ejection fraction [2]. Death from noncardiovascular causes is more common in patients who have heart failure with a preserved ejection fraction than in those with a reduced ejection fraction $[3,4]$.

HF with preserved EF (HFpEF) is characterized by a normal left ventricle ejection fraction (LVEF), normal LV end-diastolic volume, and abnormal diastolic function, often with LV concentric remodeling or hypertrophy, but sometimes with normal ventricular geometry. Most authorities currently define HFpEF by LVEF $\geq 50$ percent. The dominant abnormality resides in diastole, but there are also abnormalities in systolic function, the left atrium, pulmonary vasculature, right ventricle, arteries, and skeletal muscle. However, in clinical practice, the diagnosis of HFpEF is often one of exclusion based on the finding of a normal or near normal (or "preserved") LVEF. As discussed below, HFpEF may be best defined as HF with LVEF $\geq 50$ percent and evidence of diastolic dysfunction. Despite of various therapies improve survival in patients with HF and a reduced ejection fraction, no pharmacologic therapy has been shown to effectively reduce mortality in HFpEF patients. National registers and real world evidence studies have demonstrated a crucial relevance not only to pure medical study but also it can improve strategies for policy health [1-5].

Studies and cumulative evidence have shown that there is a third group of patients with mid-range LVEF (HFmrEF; 41 to 49 percent) that seem to share features of both HFrEF and HFpEF. While there are very few prospective trial data in this cohort, some data suggest that these patients have characteristics similar to those with HFrEF. Some experts treat these patients like HFrEF, while others treat these patients as borderline HFpEF [6].

Nevertheless, the prevalence of HFpEF increases with age. Among all patients with HF in the United States, half or more have a normal or near normal LVEF. A Mayo Clinic study examined all consecutive patients hospitalized with decompensated HF from 1987 through 2001. The proportion of patients with the diagnosis of HFpEF increased over time and was significantly higher among community patients than among referral patients (55 versus 45 percent). Over the next decade (2000 through 2010), the proportion of HF patients with HFpEF continued to increase while the incidence of HFpEF and HF with reduced EF declined. Additional data from the ARIC study show that HFpEF is by far the dominant form of HF among older adults in the United States, representing 65 to 77 percent of prevalent cases. This study also showed that the majority of adults in the community have either risk factors for HF (Stage A, 52 percent) or structural remodeling (Stage B HF, 30 percent) and are thus at increased risk for developing symptomatic HF (Stage C HF, 13 percent) [7-9].

Echocardiography is a key component of the diagnosis as well as evaluation of patients with suspected HFpEF. Echocardiography is helpful in demonstrating that the LVEF and left ventricular volume are normal. Other features that may be identified include left ventricular hypertrophy or concentric remodeling, left atrial enlargement, and evidence of diastolic dysfunction. Elevation in the pulmonary artery systolic pressure (PASP) estimated by echocardiography is very common in patients with $\mathrm{HFpEF}$, and the identification of an elevated PASP in an older patient with dyspnea should trigger consideration for the diagnosis of HFpEF $[10,11]$. 
The fundamental pathophysiological perturbation leading to heart failure with a preserved ejection fraction remains incompletely defined, but traditionally it has been attributed to hypertensive left ventricular remodeling [12] (Figure 1). Systemic microvascular endothelial inflammation related to coexisting conditions has been proposed as an additional mechanism leading to myocardial inflammation and fibrosis, increases in oxidative stress, and alterations in cardiomyocyte signaling pathways. These alterations promote cardiomyocyte remodeling and dysfunction (Figure 1) as well as microvascular dysfunction and rarefaction in cardiac and skeletal muscle [13].

Since signs and symptoms of heart failure are nonspecific, clinicians should maintain a high index of suspicion for heart failure in patients with risk factors, but they also should consider alternative or contributing diagnoses. The clinical history should include ascertainment of reduced symptoms in response to diuretic therapy and previous hospitalizations for or complicated by heart failure. In some patients, heart failure manifests as "unexplained" exertional dyspnea. In such patients, differentiating heart failure from noncardiac dyspnea or deconditioning can be challenging. In patients with suspected heart failure, comprehensive Doppler echocardiography should be performed. The relationship between pressure-volume into the left ventricle is keystone of this disease (Figure 2).

Mexico has a high prevalence of cardiovascular risk factors in adults such as hypertension (30\%), dyslipidemia (36\%), overweight and obesity (75\%) and diabetes $(12 \%)$. All of these risk factors are associated with a major risk to have HFpEF [14]. This study was focused on the description of a cohort of Mexican patients with HFpEF and to know its relationship with cardiovascular risk factors.

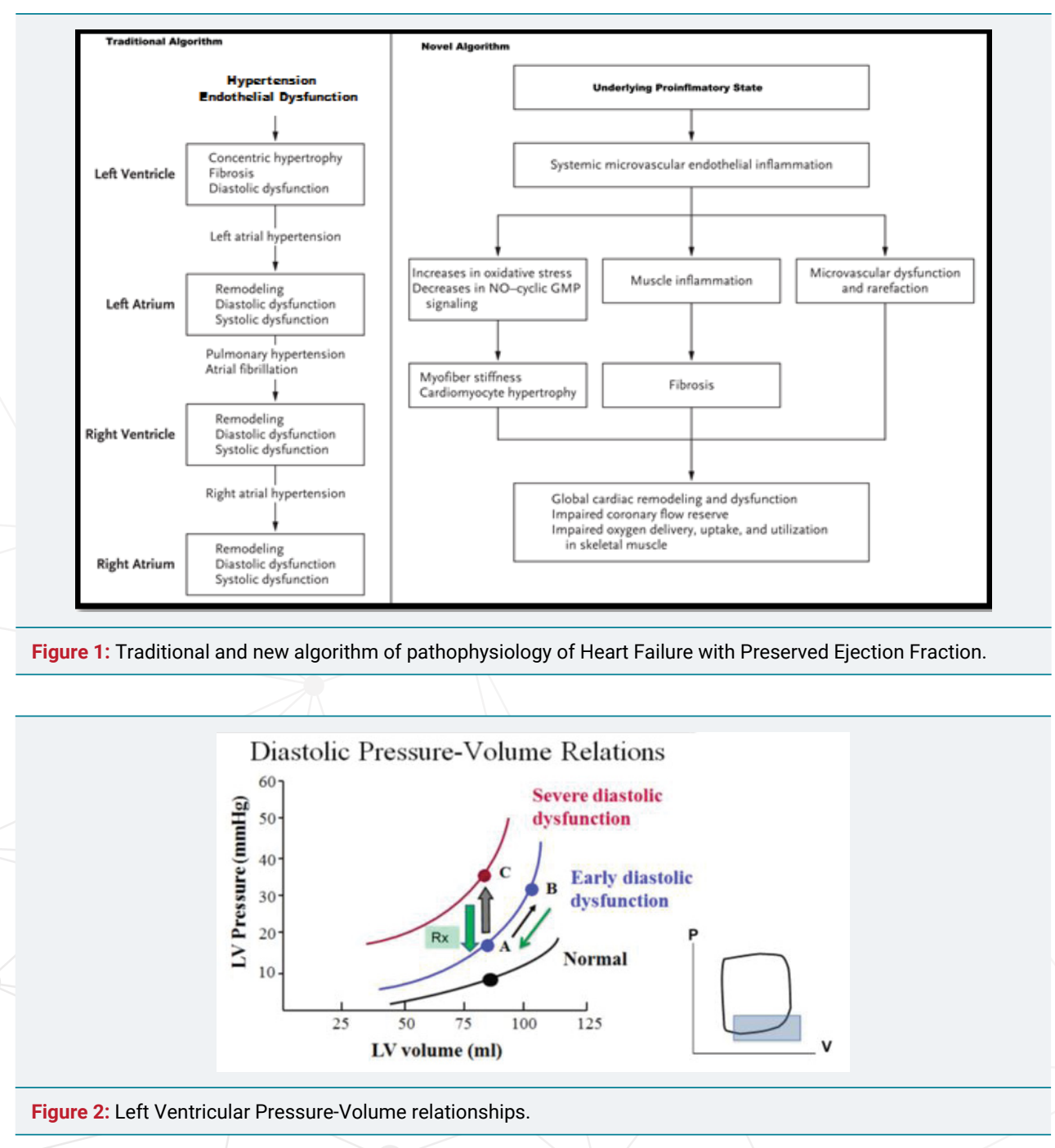




\section{Methods}

We included 168 patients from different hospitals in the metropolitan area of the Mexico City, who were sent for realization of Echocardiogram as part of their study protocol by dyspnea, edema or suspicion of hypertensive heart disease. All patients were evaluated in the Department of echocardiography at the Cardiology hospital of the CMN SXXI. Adults over 20 years old of both sexes were included. We investigate all cardiovascular risk factors known as arterial hypertension, diabetes, Dyslipidemia, and obesity. All signed informed consent we measured also levels of brain natriuretic peptide (BNP) in blood. ( ${ }^{\circledR}$ Roche Labs,) A brief medical history and physical examination was performed. Patients with any known advanced comorbidity that threaten life or give false positive of BNP in serum were not included. Those patients with very bad window for Echocardiogram were eliminated. A complete study of two-dimensional and Doppler echocardiography, including ratio E/A, deceleration time, E/e', atrial volume and morphology and mobility of the LV were recorded. The resulting annular velocities by pulsed wave Doppler were recorded for 3 to 5 cardiac cycles at a sweep speed of $100 \mathrm{~mm} / \mathrm{s}$. Measurements were performed on computerized off-line analysis stations without knowledge of invasively derived hemodynamic data. LV volumes, $\mathrm{EF}$, and left atrial (LA) maximal volume were measured as recently recommended. Mitral annulus early diastolic velocity (e0) and late diastolic velocity were measured at septal and lateral mitral annulus and $\mathrm{E} / \mathrm{e}^{\prime}$ ratios were computed. Diastolic degrees are showed in figure 3.

We also excluded patients with chronic kidney disease (GFR $<30 \mathrm{ml} / \mathrm{min}$ )), valve heart disease with criteria of severity, any comorbidity disease affecting survival at 5 years, congenital heart diseases, alleviates pressure pulmonary artery $>60 \mathrm{mmHg}$, heart disease ischemic, and non-acceptance of informed consent.

\section{Results}

General characteristics of study population are shown in table 1. As other reports female dominated this group of patients, the average age was over 60 years old and majority had hypertension or diabetes. The most common symptom was dyspnea following by edema. Notably overweight and obesity were common ( $>75 \%)$.

\section{Echocardiographic profile}

From the total population, $42.9 \%$ were diabetic and $89 \%$ had hypertension. Most of them corresponded to the female sex. According to figure 2, 69 (41.1\%) patients corresponded to Grade I of diastolic dysfunction; 96 (57.1\%) shown grade II and only 3 patients $(1.8 \%)$ were classified as grade III. Cuando estos grados de severidad de disfunción diastólica se evaluaron de acuerdo con el factor de riesgo cardiovascular

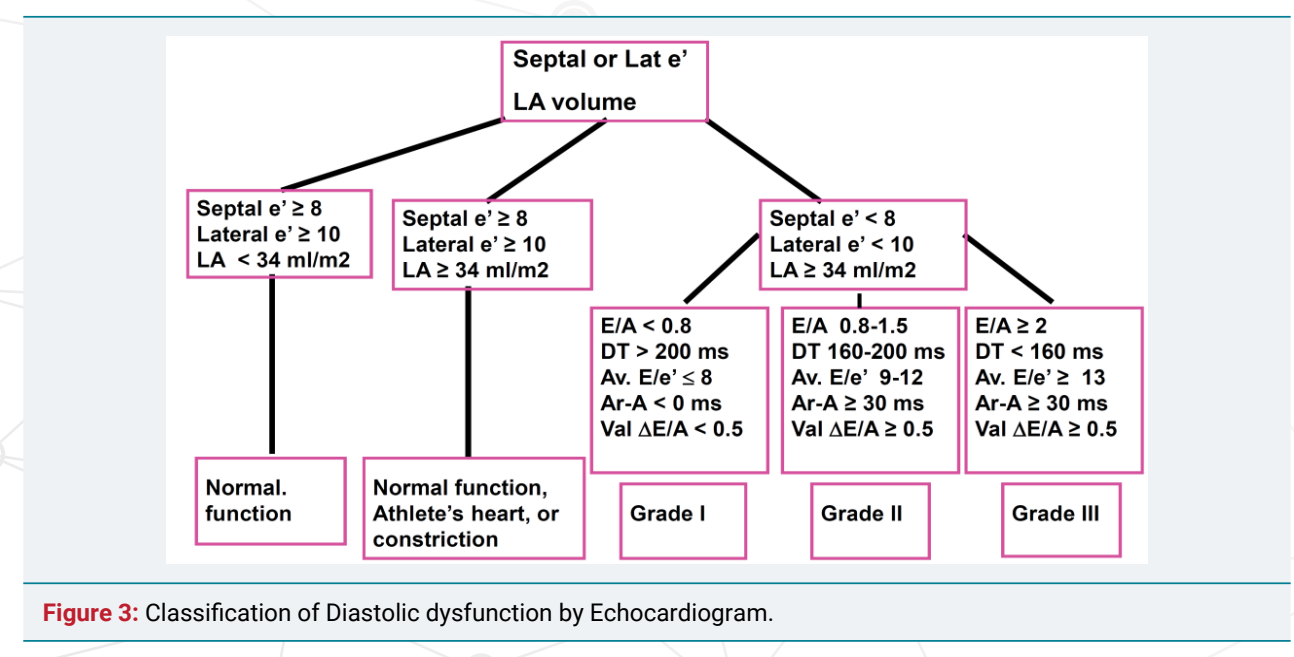


se encontró: Ser diabético se asoció a mayor severidad de DD (34\% and $66 \%$ vs nodiabetics $46 \%$ and $54 \%$ for grade I and II, respectively). Hypertension was the most important and frequent cardiovascular risk factor.

Table 2 details the Echocardiogram findings. 114 patients (67.8\%) were classified as type 1 diastolic pattern based on the E/A ratio; 54 patients were classified as type II and the pattern type III was not documented in this study. As expected Wave A was notably higher than type II, however Isovolumic relaxation time (msec) was shorter in type II than type I.

PCW was increasing as the severity of diastolic dysfunction was detected. Left atrium and global geometry of left ventricle was similar. Despite of non-statistical significance, E/e' was higher in type II than type I, and the cutoff point to separate Type I of Type II were E/e' lateral of 7.5 and 10.5 for septal E/e' (Table 2). Grade II was more common in Type II pattern independently of cardiovascular risk factors.

\section{Discussion}

This study revealed for the first time that the prevalence of HFpEF in the Mexican population is more common than we expected. We observed a higher prevalence of HFpEF in women than that in men $(64.3 \%$ vs. $35.7 \%$, respectively). The participants with female gender, hypertension, or history of heart disease tended to have a higher prevalence of HFpEF.

With the variation of the definition and diagnostic criteria of heart failure, the prevalence of HFpEF varied greatly in different studies [14-16]. The differences of study population, geographic area, and the year of data collection may also contribute to the prevalence of HFpEF [15]. A population-based cohort study found that the patients with heart failure comprised approximately $5 \%$ of the total population [17], and about half of the patients with heart failure had preserved normal left ventricular ejection fraction. Our results were in accordance with these findings. However, because the prevalence of HFpEF in general population in Mexico is not reported, we could not determine whether our results agreed with the prevalence of HFpEF in other regions worldwide.

\begin{tabular}{|c|c|}
\hline Age (years) & $63.50 \pm 9.5$ \\
\hline Female n(\%) & 108(64.3) \\
\hline Male n(\%) & $60(35.7)$ \\
\hline Weight kg & $74.68 \pm 13.77$ \\
\hline Height, $\mathrm{cm}$ & $158.4 \pm 9.9$ \\
\hline BMI (kg/m2) & $29.7 \pm 4.3$ \\
\hline$\cdot<25 \mathrm{Kg} / \mathrm{m} 2$ & $36(21.1)$ \\
\hline$\cdot 25-29-9 \mathrm{Kg} / \mathrm{m} 2$ & $81(48.2)$ \\
\hline$\cdot 30-39.9 \mathrm{Kg} / \mathrm{m} 2$ & $42(25.0)$ \\
\hline 40 or more & $09(05.3)$ \\
\hline Dyspnea & 147(87.1) \\
\hline Edema & $57(33.9)$ \\
\hline DM & $72(42.9)$ \\
\hline Hypertension & $150(89.3)$ \\
\hline Smoking & $54(32.1)$ \\
\hline Previous Stroke & $06(3.5)$ \\
\hline COPD & $09(5.3)$ \\
\hline Glucose $(\mathrm{mg} / \mathrm{dl})$ & $118.0 \pm 49.2$ \\
\hline Urea (mg/dl) & $36.3 \pm 10.4$ \\
\hline Creatinine (mg/dl) & $0.88 \pm 0.23$ \\
\hline GFR (ml/min) & $78.7 \pm 16.4$ \\
\hline $\mathrm{BNP}(\mathrm{pg} / \mathrm{ml})$ & $220.7 \pm 328.5$ \\
\hline
\end{tabular}


Table 2: Echocardiographic profile by type of diastolic dysfunction pattern

\begin{tabular}{|c|c|c|c|}
\hline \multirow{2}{*}{ Parameter } & \multicolumn{2}{|c|}{ Diastolic Dysfunction Pattern } & \multirow{2}{*}{ p Value } \\
\hline & Type I $(n=114)$ & Type II $(n=54)$ & \\
\hline $\mathrm{LAV}(\mathrm{ml} / \mathrm{m} 2)$ & $29.2 \pm 10.18$ & $31.8 \pm 19.9$ & 0.101 \\
\hline E wave $(\mathrm{cm} / \mathrm{sec})$ & $63.5 \pm 15.20$ & $82.17 \pm 17.63$ & 0.397 \\
\hline A Wave $(\mathrm{cm} / \mathrm{sec})$ & $88.8 \pm 18.03$ & $67.93 \pm 13.93$ & 0.060 \\
\hline E/A index $(\mathrm{cm} / \mathrm{sec})$ & $0.72 \pm 0.14$ & $1.21 \pm 0.19$ & 0.010 \\
\hline Deceleration time (msec) & $249.50 \pm 48.94$ & $215.04 \pm 33.81$ & 0.007 \\
\hline Isovolumic relaxation time (msec) & $113.30 \pm 19.43$ & $103.87 \pm 20.60$ & 0.369 \\
\hline $\operatorname{LVEF}(\%)$ & $66.19 \pm 6.20$ & $66.81 \pm 6.90$ & 0.297 \\
\hline LV, final systolic volume (ml) & $26.03 \pm 10.11$ & $23.55 \pm 6.42$ & 0.004 \\
\hline SPPA $(\mathrm{mmHg})$ & $32.25 \pm 7.44$ & $35.37 \pm 9.56$ & 0.018 \\
\hline PCW $(\mathrm{mmHg})$ & $11.38 \pm 3.36$ & $13.93 \pm 3.29$ & 0.744 \\
\hline DDLV (mm) & $41.60 \pm 6.10$ & $42.29 \pm 4.88$ & 0.330 \\
\hline $\operatorname{SDLV}(\mathrm{mm})$ & $26.54 \pm 4.92$ & $26.13 \pm 3.50$ & 0.058 \\
\hline Septum (mm) & $11.59 \pm 2.30$ & $11.81 \pm 2.43$ & 0.900 \\
\hline Posterior wall (mm) & $11.54 \pm 2.07$ & $11.48 \pm 2.08$ & 0.531 \\
\hline TAPSE (mm) & $21.04 \pm 3.31$ & $21.19 \pm 2.82$ & 0.386 \\
\hline MR n(\%) & $60(52.6)$ & $33(61.1)$ & 0.302 \\
\hline TR n(\%) & $90(78.9)$ & $48(88.9)$ & 0.085 \\
\hline AoR n(\%) & $31(27.2)$ & $11(20.4)$ & 0.224 \\
\hline PR n(\%) & $06(05.3)$ & $06(11.1)$ & 0.146 \\
\hline IVC (mm) & $14.58 \pm 2.80$ & $14.89 \pm 2.71$ & 0.556 \\
\hline LVMI (g/m2) & $104.80 \pm 24.80$ & $105.28 \pm 18.77$ & 0.053 \\
\hline $\mathrm{e}^{\prime}$ lateral $(\mathrm{cm} / \mathrm{sec})$ & $08.92 \pm 2.47$ & $8.78 \pm 2.06$ & 0.173 \\
\hline E/e'Lateral & $7.58 \pm 2.60$ & $9.66 \pm 2.60$ & 0.759 \\
\hline $\mathrm{e}^{\prime}$ septal $(\mathrm{cm} / \mathrm{sec})$ & $6.16 \pm 1.53$ & $6.31 \pm 1.62$ & 0.884 \\
\hline E/e' Septal & $10.62 \pm 2.97$ & $13.26 \pm 3.76$ & 0.263 \\
\hline
\end{tabular}

LAV, Left atrial volume; LVEF, Left Ventricle Ejection Fraction; SPPA, systolic pressure of pulmonary artery; PCW, pulmonary capillary wedge; DDLV, diastolic diameter of left ventricle; SDLV, systolic diameter of left ventricle; TAPSE, Tricuspid annular plane systolic excursion; MR, mitral regurgitation; TR, tricuspid regurgitation; AoR, Aortic regurgitation; PR, pulmonary regurgitation; IVC, inferior cava venous; LVMI, left ventricle mass index; Type 1, relaxation disorder; Type 2, Pseudo-normalization pattern.

In general, most studies have concluded that age is an important risk factor for $\mathrm{HFpEF}$ [3]. We found that the incidental cases of HFpEF were found at mean age of 63 years old, which was consistent with the result of a study conducted in other countries [15].

Nevertheless, we identified a very high rate of hypertension, overweight or obesity and Dyslipidemia. Diabetes was also highly prevalent. In Mexico the cardiovascular risk factors prevalence rate is higher in absolute numbers in population aged $<54$ years, therefore we think that for when the symptoms of heart failure appears, It have passed at least 10 or 15 years of the onset of this chronic risk factors.

Regarding gender, in the present study, we found that women had a higher prevalence of HFpEF, which was in accordance with another study that found the age-standardized prevalence of HFpEF for women and men was $64.3 \%$ and $35.7 \%$, respectively. The Rotterdam Study reported that the overall prevalence of heart failure was $3.9 \%$ and did not differ between men and women, but the prevalence of left ventricular systolic dysfunction (HFREF) was higher in men than in women [16-19]. Frank P. Brouwers et al. [20], found that female gender, atrial fibrillation, higher cystatin $\mathrm{C}$, and urinary albumin excretion were particularly strong predictors for HFpEF. This is possibly because women are more likely to suffer from metabolic syndrome, which is characterized by hyperlipidemia, hypertension, diabetes mellitus, abdominal obesity [21], and stroke [22]. These diseases could increase the risk of HFpEF in women, as previously mentioned.

Our study found that participants with clinical comorbidities such as hypertension, dyslipidemia, obesity, and smoking had a higher association with HFpEF, consistent with the results from other studies [21-23]. Of note, renal dysfunction and cardiorenal syndrome were uncommon in patients with $\mathrm{HFpEF}$, which might be related to protracted fluid retention and refractory hypertension [21]. Unlike the unalterable 
factors, such as age and gender, an appropriate treatment of the comorbidity could be crucial for the prevention of HFpEF. For example, hypertension is generally considered to lead to the development of HFpEF [23], which is consistent with our results. Hence, early diagnosis and treatment of hypertension was proven to be effective for the prevention of HFpEF.

In our study, Brain natriuretic peptide (BNP) or was checked and important findings were detected. As the ejection fraction was lower and closer to $45 \%$ higher levels of BNP were detected (Figure 4).

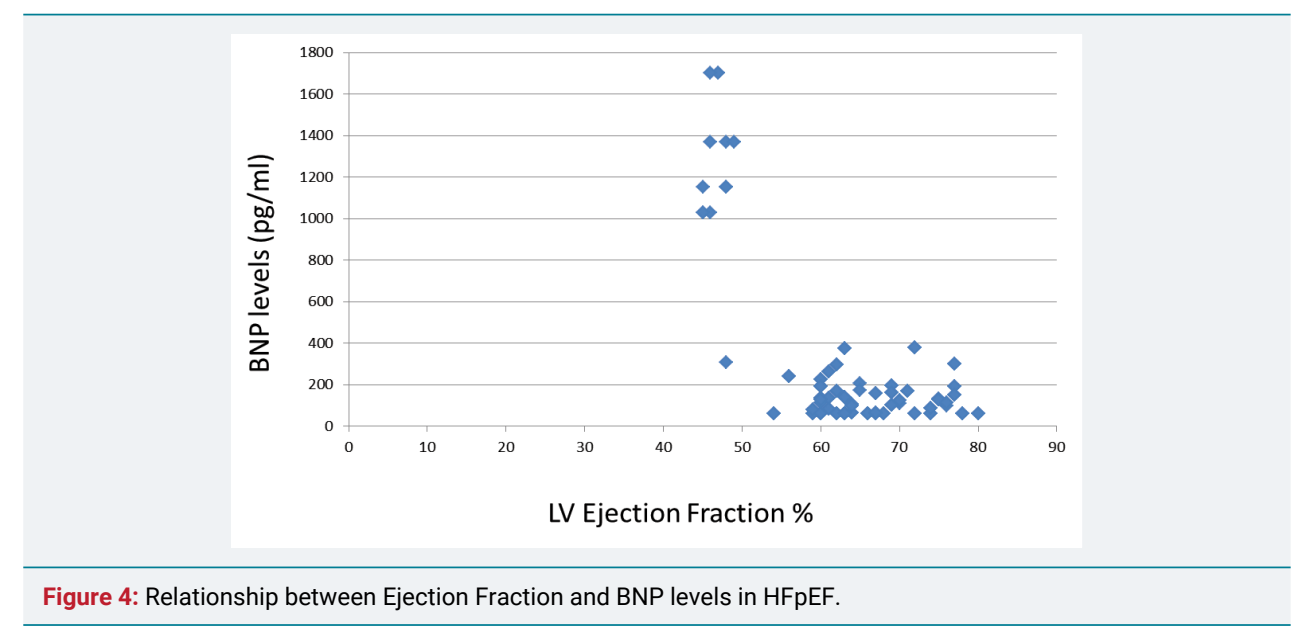

\section{Conclusion}

HFpEF is a frequent entity in patients with cardiovascular risk factors in Mexico. The most common risk factor was hypertension. The combination of hypertension, overweight and dyslipidemia predicted the severity of diastolic dysfunction. We recommend that all Mexican patient with hypertension and overweight or obesity should be submitted as a part of its medical evaluation to an echocardiogram study in order to detect diastolic dysfunction even though the signs or symptoms are or not evident.

\section{References}

1. Redfield MM. Heart Failure with Preserved Ejection Fraction. N Engl J Med. 2016; 375: 1868-1877. Ref.: https://goo.gl/15NsQA

2. Verma A, Solomon SD. Diastolic dysfunction as a link between hypertension and heart failure. Med Clin North Am. 2009; 93: 647-664. Ref.: https://goo.gl/VMPuz6

3. Gladden JD, Linke WA, Redfield MM. Heart failure with preserved ejection fraction. Pflugers Arch. 2014; 466: 1037-1053. Ref.: https://goo.gl/p8Z9Bd

4. Campbell RT, Jhund PS, Castagno D, Hawkins NM, Petrie MC, et al. What have we learned about patients with heart failure and preserved ejection fraction from DIG-PEF, CHARM-preserved, and I-PRESERVE? J Am Coll Cardiol. 2012; 60: 2349-2356. Ref.: https://goo.gl/uqKLRy

5. Wan SH, Vogel MW, Chen HH. Pre-clinical diastolic dysfunction. J Am Coll Cardiol. 2014; 63: 407416. Ref.: https://goo.gl/9jiHrd

6. Redfield MM, Jacobsen SJ, Burnett JC Jr, Mahoney DW, Bailey KR, et al. Burden of systolic and diastolic ventricular dysfunction in the community: appreciating the scope of the heart failure epidemic. JAMA 2003; 289: 194-202. Ref.: https://goo.gl/rydHDx

7. Chobanian AV, Bakris GL, Black HR, Cushman WC, Green LA, et al. Seventh report of the Joint National Committee on Prevention, Detection, Evaluation, and Treatment of High Blood Pressure. Hypertension. 2003; 42: 1206-1252. Ref.: https://goo.gl/xMHwb5

8. Shah SJ, Kitzman DW, Borlaug BA, van Heerebeek L, Zile MR, et al. Phenotype-specific treatment of heart failure with preserved ejection fraction: a multiorgan roadmap. Circulation. 2016; 134: 73-90. Ref.: https://goo.gl/P1Weyv 
9. Owan TE, Hodge DO, Herges RM, Jacobsen SJ, Roger VL, et al. Trends in prevalence and outcome of heart failure with preserved ejection fraction. N Engl J Med. 2006; 355: 251-259. Ref.: https://goo.gl/1NRJv7

10. Nagueh SF. Classification of Left Ventricular Diastolic Dysfunction and Heart Failure Diagnosis and Prognosis. J Soc Am Echo. 2018; 31: 1209-1211. Ref.: https://goo.gl/qrytM8

11. Nagueh SF, Smiseth OA, Appleton CP, Byrd BF III, Dokainish H, et al. Recommendations for the evaluation of left ventricular diastolic function by echocardiography: an update from the American Society of Echocardiography and the European Association of Cardiovascular Imaging. J Am Soc Echocardiogr. 2016; 29: 277-314. Ref.: https://goo.gl/XKNctv

12. Zile MR, Brutsaert DL. New concepts in diastolic dysfunction and diastolic heart failure: Part II: causal mechanisms and treatment. Circulation. 2002; 105: 1503-1508. Ref.: https://goo.gl/55jYXc

13. Morgan JP. Abnormal intracellular modulation of calcium as a major cause of cardiac contractile dysfunction. N Engl J Med. 1991; 325: 625-632. Ref.: https://goo.gl/QACwLk

14. Rosas-Peralta M, Borrayo-Sánchez G, Ramírez-Arias E, Jiménez-Genchi GM, Hernández-González MA, et al. Cardiovascular Risk Reduction: Past Present and Future in Mexico. Ann Clin Hypertens. 2018; 2: 038-047. Ref.: https://goo.gl/BGCk7V

15. Chan MM, Lam CS. How do patients with heart failure with preserved ejection fraction die? Eur J Heart Fail. 2013; 15: 604-613. Ref.: https://goo.gl/sL9itT

16. Lee DS, Gona P, Albano I, Larson MG, Benjamin EJ, et al. A systematic assessment of causes of death after heart failure onset in the community: impact of age at death, time period, and left ventricular systolic dysfunction. Circ Heart Fail. 2011; 4: 36-43. Ref.: https://goo.gl/C1RHx8

17. Nadruz W, Shah AM, Solomon SD. Diastolic Dysfunction and Hypertension. Med Clin N Am. 2017; 101: 7-17. Ref.: https://goo.gl/sd3RX3

18. Wan SH, Vogel MW, Chen HH. Preclinical Diastolic Dysfunction. J Am Coll Cardiol. 2014; 11; 63: 407-416. Ref.: https://goo.gl/uxU6mi

19. Mosterd A, Hoes AW, de Bruyne MC, Deckers JW, Linker DT, et al. Prevalence of heart failure and left ventricular dysfunction in the general population; The Rotterdam Study. Eur Heart J. 1999; 20: 447-455. Ref.: https://goo.gl/fq5Lp6

20. Brouwers FP, de Boer RA, van der Harst P, Voors AA, Gansevoort RT, et al. Incidence and epidemiology of new onset heart failure with preserved vs. reduced ejection fraction in a community-based cohort: 11-year follow-up of PREVEND. Eur Heart J 2013; 34: 1424-1431. Ref.: https://goo.gl/JxtcNa

21. Tsutsui $H$, Tsuchihashi $M$, Takeshita A. Mortality and readmission of hospitalized patients with congestive heart failure and preserved versus depressed systolic function. Am J Cardiol. 2001; 88: 530-533. Ref.:

22. Lam CS, Carson PE, Anand IS, Rector TS, Kuskowski M, et al. Sex differences in clinical characteristics and outcomes in elderly patients with heart failure and preserved ejection fraction: The Irbesartan in Heart Failure with Preserved Ejection Fraction (I-PRESERVE) trial. Circ Heart Fail. 2012; 5: 571-578. Ref.: https://goo.gl/g8dBnm

23. Kälsch H, Lehmann N, Möhlenkamp S, Neumann T, Slomiany U, et al. Association of coronary artery calcium and congestive heart failure in the general population: Results of the Heinz Nixdorf Recall study. Clin Res Cardiol. 2010; 99: 175-182. Ref.: https://goo.gl/H4th8M 